\title{
Changes in electrophysiological properties of photoreceptors in Periplaneta americana associated with the loss of screening pigment
}

Paulus Saari ${ }^{1}$, Esa-Ville Immonen ${ }^{1}$, Joni Kemppainen ${ }^{1}$, Kyösti Heimonen ${ }^{1}$, Marianna Zhukovskaya ${ }^{2}$, Ekaterina Novikova ${ }^{2}$, Andrew S. French ${ }^{3}$, Päivi H. Torkkeli ${ }^{3}$, Hongxia Liu ${ }^{3}$, and Roman V. Frolov ${ }^{1}$ ${ }^{1}$ Biophysics group, Nano and Molecular Systems Research Unit, University of Oulu, Oulu, Finland ${ }^{2}$ Sechenov Institute of Evolutionary Physiology and Biochemistry, Russian Academy of Sciences, 44 Thorez Pr., Saint Petersburg 194223, Russia

${ }^{3}$ Department of Physiology and Biophysics, Dalhousie University, P.O. BOX 15000, Halifax, NS, B3H 4R2, Canada

Corresponding author: Roman V. Frolov. Address: Biophysics group, Nano and Molecular Systems Research Unit, University of Oulu, Oulu, Finland; ORCID 0000-0002-7431-5297; e-mail: roman.frolov@oulu.fi.

\section{Funding}

The work was supported by State budget grant of Russian Federation \#AAAA-A18-118013090245-6 to MZ and Natural Sciences and Engineering Research Council of Canada (NSERC) grants RGPIN/05565 (to P. H. Torkkeli) and RGPIN/03712 (to A. S. French). 


\begin{abstract}
Absence of screening pigment in insect compound eyes has been linked to visual dysfunction. We investigated how its loss in a white-eyed mutant (W-E) alters the photoreceptor electrophysiological properties, opsin gene expression and the behavior of the cockroach, Periplaneta americana. Whole-cell patch-clamp recordings of green-sensitive photoreceptors in W-E cockroaches gave reduced membrane capacitance, absolute sensitivity to light and light-induced currents. Decreased low-pass filtering increased voltage bump amplitudes in W-E photoreceptors. Intracellular recordings showed that angular sensitivity of W-E photoreceptors had two distinct components: a large narrow component with the same acceptance angle as wild-type, plus a relatively small wide component. Information processing was evaluated using Gaussian white-noise modulated light stimulation. In bright light, W-E photoreceptors demonstrated higher signal gain and signal power than wild-type photoreceptors. Expression levels of the primary UV- and green-sensitive opsins were lower and of the secondary green-sensitive opsin significantly higher in W-E than in wild-type retinae. In behaviour experiments, W-E cockroaches were significantly less active in dim green light, consistent with the relatively low light sensitivity of their photoreceptors. Overall, these differences can be related to the loss of screening pigment function and to a compensatory decrease in the rhabdomere size in W-E retinae.
\end{abstract}

Keywords: compound eye, white-eyed, screening pigment, photoreceptor, Periplaneta americana
ABBREVIATIONS:
CL, constant light
$\mathrm{CD}$, constant dark
$C_{\mathrm{m}}$, whole-cell membrane capacitance
IR, information rate
$\mathrm{Kv}$, voltage-gated $\mathrm{K}^{+}$current or conductance
LIC, light-induced current
W-E, white-eyed 


\section{Introduction}

Photostable screening pigments in arthropod compound eyes serve multiple purposes (for a comprehensive review, see (Stavenga 1989)). They set or contribute to setting the eye color. Pigment cells surrounding the photoreceptors block spread of stray light within the retina, whereas the pigment granules within the photoreceptor form a barrier between the rhabdomere and the soma that dynamically regulates the intensity of light propagating along the optical waveguide of the rhabdom. The pigment cell and granule migration is essential for photoreceptor light and dark adaptations as it adjusts the acceptance angle of the ommatidium and light absorption in the waveguide, trading acuity for absolute sensitivity in the dark and vice versa in the light (Kirschfeld and Franceschini 1969; Walcott 1971; Butler and Horridge 1973; Stavenga et al. 1977; Immonen et al. 2014). In fly photoreceptors, these mechanisms can alter the light flux by 2-3 orders of magnitude and thus strongly extend the functional range of photoreceptors (Howard et al. 1987). In many arthropod eyes, screening pigments can selectively absorb short-wavelength light while transmitting long-wavelengths, thus sharpening and shifting photoreceptor spectral sensitivity (Lall et al. 1988; Arikawa et al. 1999; Arikawa et al. 2009). In flies, screening pigment facilitates metarhodopsin photoregeneration into rhodopsin (Stavenga et al. 1973) and can also protect the visual pigment from destruction by intense ultraviolet (UV) light (Zhu and Kirschfeld 1984).

Given the importance of screening pigments, it is likely that their absence will severely compromise photoreceptor functions. Studies of white-eyed and albino mutants in several insect species support this hypothesis; in white-eyed honeybees and blowflies, visual fields were broadened and the photoreceptor contrast detection capacity was dramatically decreased, especially in bright light (Streck 1972; Howard et al. 1987; Gribakin 1988; Gribakin et al. 1996). The photoreceptors of white eyed mutant (white) Drosophila melanogaster, have higher sensitivity to light and a broader intensity range of light responses than wildtype, plus an abnormal electroretinogram (ERG) (Alawi et al. 1972; Pak and Lidington 1974; Wu and Wong 1977). The white mutant has fewer facets and reduced optomotor responses compared to wild-type (Kalmus 1943). However, not all of these defects can be attributed to the loss of screening pigment, because the mutation in an $\mathrm{ABC}$ transporter underlying the white phenotype also affects other neurological functions (Borycz et al. 2008).

Screening pigments have clearly diverse function within and between species. Due to this diversity, and the fact that only relatively few studies have been performed at the cellular level (Streck 1972; Howard et al. 1987), the consequences of the absence of screening pigments on photoreceptor functioning are poorly understood. The goal of the present work was to investigate the differences in electrophysiological properties of photoreceptors of the wild-type nocturnal cockroach, Periplaneta americana, and a spontaneous white-eyed (W-E) mutant strain and to relate these finding to potential changes in the expression of opsin genes and to the cockroach behavior.

Recently, using patch-clamp experiments, we found that the whole-cell capacitance (a proxy for cell membrane area), absolute sensitivity to light, and light-induced current (LIC) amplitudes decreased 
strongly in $P$. americana photoreceptors when the animals were kept in constant light for several months, but constant dark exposure lead to an increase in the same parameters (Frolov et al. 2018). These changes suggest adaptive structural modifications in the rhabdom and are different to previous findings in $D$. melanogaster. We also observed changes in the speed of phototransduction, information processing and expression of genes involved in phototransduction.

In the present study, we hypothesized that when the white-eyed cockroaches are reared in normal lighting conditions (12 hour light: 12 hour dark) the absence of screening pigment exposes their retinae to a stronger illumination than in wild type cockroaches. This could lead to comparable changes in photoreceptor properties to those found after constant light exposure (Frolov et al. 2018). We tested this hypothesis using patch-clamp recordings from photoreceptors in dissociated ommatidia and in vivo intracellular recordings. Compared to wild-type, W-E cockroaches were characterized by smaller wholecell membrane capacitance, lower absolute sensitivity and light-induced current amplitudes, increased voltage bumps, and anomalous angular sensitivity. Consistently, expression of the major opsins decreased.

\section{Materials and Methods}

\section{Animals}

Wild-type Periplaneta americana were obtained from different sources: purchased from Blades Biological (Blades Biological Ltd, Edenbridge, Kent, UK), or from laboratory colonies at Dalhousie and Oulu universities. White-eyed (W-E) cockroaches originate from the pearl strain (Ross et al. 1964) and reared in a laboratory colony for several generations. Cockroaches used in electrophysiological and behavioral experiments were maintained in reversed $12 \mathrm{~h}$ light: $12 \mathrm{~h}$ dark illumination conditions with plenty of shelter from light available. An in-built incubator light source provided ca. 600 lux illumination during the day phase of the cycle. Only adult male cockroaches were used in experiments.

\section{Quantitative polymerase chain reaction analysis}

mRNA concentrations were measured by real-time quantitative polymerase chain reaction (PCR) as described previously (French et al. 2015). In brief, total RNA was extracted from 14 retinas of wild-type and 16 retinas of white-eyed $P$. americana using a RNeasy Plus mini kit (Qiagen, Valencia, CA, USA) and evaluated using an Experion RNA Analysis Kit (Bio-Rad, Mississauga, Ontario, Canada) after treatment with RNase-free DNase I (Ambion). $50 \mathrm{ng}$ of total RNA was used for first-strand cDNA synthesis with ProtoScript II reverse transcriptase (New England BioLabs). Quantitative PCR was performed using GoTaq qPCR Master (Promega, Madison, WI, USA) on a CFX96TM real-time PCR detection system (Bio-Rad) as described previously (Immonen et al. 2017). All PCR runs were repeated three times. Gene expression levels, PCR efficiency, and the standard error of measurement were calculated using CFX Manager (BioRad). The primer sequences for the specific and reference genes are provided elsewhere (French et al. 2015). Amplification efficiencies of the primers were determined using serially diluted cDNA samples. 


\section{Patch-clamp recordings}

Ommatidia were dissociated and whole-cell recordings were performed as described previously (Immonen et al. 2017). In brief, data were acquired using an Axopatch1-D patch-clamp amplifier, Digidata1550 digitizer and pClamp10 software (Axon Instruments/Molecular Devices, CA, USA). Patch electrodes were made from thin-walled borosilicate glass (World Precision Instruments, Sarasota, FL, USA). Electrode resistances were between 3 and $9 \mathrm{M} \Omega$. Bath solution contained (in $\mathrm{mM}$ ): $120 \mathrm{NaCl}, 5 \mathrm{KCl}, 4 \mathrm{MgCl}_{2}, 1.5$ $\mathrm{CaCl}_{2}, 10 \mathrm{~N}$-Tris-(hydroxymethyl)-methyl-2-amino-ethanesulfoncic acid (TES), 25 proline and 5 alanine, $\mathrm{pH}$ 7.15. Patch pipette solution contained (in $\mathrm{mM}$ ): $100 \mathrm{~K}$-gluconate, $40 \mathrm{KCl}, 10 \mathrm{TES}, 2 \mathrm{MgCl}_{2}, 4 \mathrm{Mg}$ ATP, $0.4 \mathrm{Na}-\mathrm{GTP}$ and $1 \mathrm{NAD}, \mathrm{pH}$ 7.15. The liquid junction potential (LJP) was $-12 \mathrm{mV}$. All voltage values cited in the text were corrected for the LJP. The series resistance was compensated by $80 \%$ and typically did not exceed $10 \mathrm{M} \Omega$ during voltage-clamp recordings. Membrane capacitance was calculated from the total charge flowing during capacitive transients for voltage steps from -112 to $-92 /-82 \mathrm{mV}$.

\section{Intracellular recordings}

In vivo intracellular single-electrode recordings were performed as described previously (Saari et al. 2017). In brief, the dorsal part of the left compound eye was used in the experiments. Photoreceptor responses were recorded using microelectrodes (borosilicate glass; Harvard Apparatus) manufactured with a laser puller (P-2000; Sutter Instrument) and filled with $2 \mathrm{M} \mathrm{KCl}$ solution, $\mathrm{pH} 6.84$, to a final resistance of 100$150 \mathrm{M} \Omega$. The reference electrode was placed through the left antenna. Signals were recorded with a singleelectrode amplifier (SEC-05L; NPI).

\section{Light stimulation during electrophysiological measurements}

Light stimulation was performed as described previously (Saari et al. 2017). In brief, a custom-made voltage-to-current converter for light-emitting diodes (LEDs) was used to drive 10 (in patch-clamp experiments) or 14 (in intracellular experiments) LEDs (Roithner Laser Technik, Austria), with narrowband emission peaks ranging from 355 to $625 \mathrm{~nm}(355,385,400,435,450,462,490,505,525,545,572$, $594,612,625 \mathrm{~nm}$ ), which were used in combination with a series of neutral density (ND) filters (Kodak, New York, NY, USA). Light intensities are presented as dimensionless values in the range from $5 \cdot 10^{-6}$ to 10; the numbers correspond to the voltage range driving the voltage-to-current converter, with attenuation by ND filters taken into account. In patch-clamp experiments the spectral class of photoreceptors was determined using a simple protocol consisting of $20 \mathrm{~ms}$ isoquantal flashes of light from all ten LEDs at an intermediate light intensity.

In absolute sensitivity measurements, to minimize errors due to photoreceptor activation by the residual light background, the stimulus intensity was adjusted by using ND filters so as to elicit 1 to 10 bumps $\mathrm{s}^{-1}$. To enable comparison of photoreceptors stimulated at different ND filters, bump rates were recalculated in the following manner. If a wild-type photoreceptor gave 1 bump s$^{-1}$ at ND filter \#6 (relative light intensity of $5 \cdot 10^{-6}$ ) on average, and a photoreceptor in a W-E animal produced 1 bump s ${ }^{-1}$ at ND filter \#5 (relative light intensity of $5 \cdot 10^{-5}$ ), we concluded that the latter is ten times less sensitive than the former. 
To plot all values at the same light intensity, we assigned the sensitivity of the former cell at 1 and the latter at 0.1 bump s$^{-1}$. Such procedure was performed for all photoreceptors with relatively low or high absolute sensitivities.

\section{Angular sensitivity}

The optical axis of the photoreceptor was determined by changing the polar and azimuthal angles of the light source while recording light responses, using a Cardan-arm system. In this on-axis position, the photoreceptor was stimulated by $20 \mathrm{~ms}$ flashes delivered in $10^{0.5}$ increments over a $10^{4}$ intensity range with the LED to which the photoreceptor was most sensitive. These responses were used to derive a $V$ $\log \left(I / I_{0}\right)$ function. To measure angular sensitivity, the polar angle was varied in 1 to 3 degree increments while recording responses to isoquantal $20 \mathrm{~ms}$ stimuli of an intermediate intensity that elicited voltage responses with maximal peak depolarization of below $25 \mathrm{mV}$. Recordings in each photoreceptor were performed at least twice, in opposite directions, with responses averaged. After correcting the polar angle values for the azimuthal angle, sensitivity values were found by first fitting the $V-\log \left(I / I_{0}\right)$ function with the Hill equation, giving the $V_{\max }$ and Hill coefficient $N$ values. Sensitivity coefficients $C_{\theta}$ at each corrected polar angle $\theta$ were then calculated using equation

$$
C_{\theta}=\left(\frac{V_{\theta} / V_{\max }}{1-V_{\theta} / V_{\max }}\right)^{\frac{1}{N}}
$$

where $V_{\theta}$ is the corresponding voltage response amplitude, and then normalized by dividing with the largest $C_{\theta}$, which was usually found at the optical axis, giving relative sensitivities. Angular sensitivity was measured in dark-adapted photoreceptors. The angular size of the light source was 2.5 degrees, substantially smaller than the mean acceptance angle (see Results).

\section{Analysis of information processing}

To evaluate information processing, a $61 \mathrm{~s}$ stimulus (photoreceptor input) consisting of a $1 \mathrm{~s}$ adapting steady light followed by 30 repetitions of a $2 \mathrm{~s}$ Gaussian white noise (GWN) sequence was used as described previously (Saari et al. 2017). The GWN had mean contrast of 0.36 and a $3 \mathrm{~dB}$ corner frequency of $50 \mathrm{~Hz}$. Data analysis was performed in MatLab (MathWorks, Massachusetts, USA). A 2 s signal $S(f)$ was calculated by averaging voltage responses to 30 repetitions of the GWN sequence. The noise traces were obtained by subtracting the signal from 30 original 2 s sequences and averaging the noise spectra $N(f)$. The signal gain of voltage responses $|T(f)|$ was calculated by dividing the cross-spectrum of photoreceptor input $C(f)$ and output (photoreceptor signal) $S(f) \cdot C^{*}(f)$ by the autospectrum of the input $C(f) \cdot C^{*}(f)$ and taking the absolute value of the resulting frequency response function (Stein et al. 1972):

$$
T(f)=\frac{S(f) \cdot C^{*}(f)}{C(f) \cdot C^{*}(f)}
$$


The Shannon information rate $(I R)$ was calculated from the signal-to-noise ratio (SNR) $S(f) / N(f)$ in the frequency range from 1 to $50 \mathrm{~Hz}$ (Shannon 1949):

$$
I R=\int_{1}^{50} \log _{2}\left(\frac{|S(f)|}{|N(f)|}+1\right) d f
$$

\section{Behavioral experiments}

Behavioral experiments were performed using a previously described experimental setup under the same conditions (Zhukovskaya et al. 2017). In brief, we recorded the activity of a single naive cockroach that entered an empty testing chamber $(20 \times 20 \times 8 \mathrm{~cm})$ from the living quarters where a group of 15 cockroaches had resided for at least two weeks. First, the cockroach was admitted to the testing chamber and allowed to explore in dim red light for $10 \mathrm{~min}$. This was followed by a $30 \mathrm{~min}$ video recording under the same conditions ("session 1" or S1). The cockroach then spent a further 10 minutes in red light without any recording. The second $30 \mathrm{~min}$ recording session (S2) was conducted under different illumination conditions. In control experiments, the same dim red light was used during S2 as during S1. Its light spectrum was characterized by a relatively narrow peak at $690 \mathrm{~nm}$. Video recordings were made using a VSC-756 USB high-resolution monochrome video camera (EVS, Russia). The total emitted powers measured at the opposite wall of the shelter were 5 and $10 \mu \mathrm{W} \mathrm{cm}{ }^{-2}$ for the red and green LEDs, respectively. Each cockroach was used for only one experiment. The chamber was cleaned thoroughly between the experiments.

\section{Statistics}

During statistical analysis, the Shapiro-Wilk normality test was applied to data samples to determine if they could be analyzed using parametric statistical methods. Data in the samples that did not pass the normality test were presented using medians and interquartile ranges (25\% quartile:75\% quartile). To evaluate differences between such samples, the Mann-Whitney U test (MWUT) was used. The samples that passed the normality test were analysed by parametric statistical methods as indicated. Such data are presented as mean \pm s.d. and compared using a two-tailed unpaired $t$-test with unequal variances. In Figs. $7 \mathrm{~d}$, e and $\mathrm{g}$ data are presented as median \pm median absolute deviation (m.a.d.). Spearman's rank order correlation coefficient (SROCC, $\rho$ ) was used in analyses of correlations. In figures, $\left(^{*}\right)$ indicates $P<0.05$ and (**) indicates $P<0.01$. Throughout the text $(n)$ stands for experimental group size.

\section{Results}

Recordings were performed in vivo from intact eyes in the current-clamp mode, and in vitro from dissociated photoreceptors by patch-clamp in both voltage- and current-clamp modes. The compound eye 
of $P$. americana contains two spectral types of photoreceptors, UV- and green-sensitive (Mote and Goldsmith 1970). Because of the small number of UV-sensitive photoreceptors in intracellular recordings and their extremely rare findings in patch-clamp experiments, all electrophysiological experiments reported in this study were performed on green-sensitive photoreceptors.

\section{Morphology}

Heads of typical male wild-type and W-E cockroaches are shown in Figs. $1 \mathrm{a}$ and $\mathrm{b}$, respectively. Photographs of corneal surfaces are shown in Figs. 1c and d. Figure 1e shows a micrograph of the cross-section of a wild-type ommatidium from its distal region. In this figure the eye is in the dark-adapted state and the pigment granules do not form a continuous sheath around the rhabdom. Figures if and $\mathrm{g}$ show photographs of dissociated wild-type and W-E ommatidia, respectively. Examination of W-E ommatidia revealed that the ommatidium in $P$. americana is two-tiered. The photoreceptors with relatively small bodies are situated in the distal and those with relatively large cell bodies in the proximal part of the ommatidium.

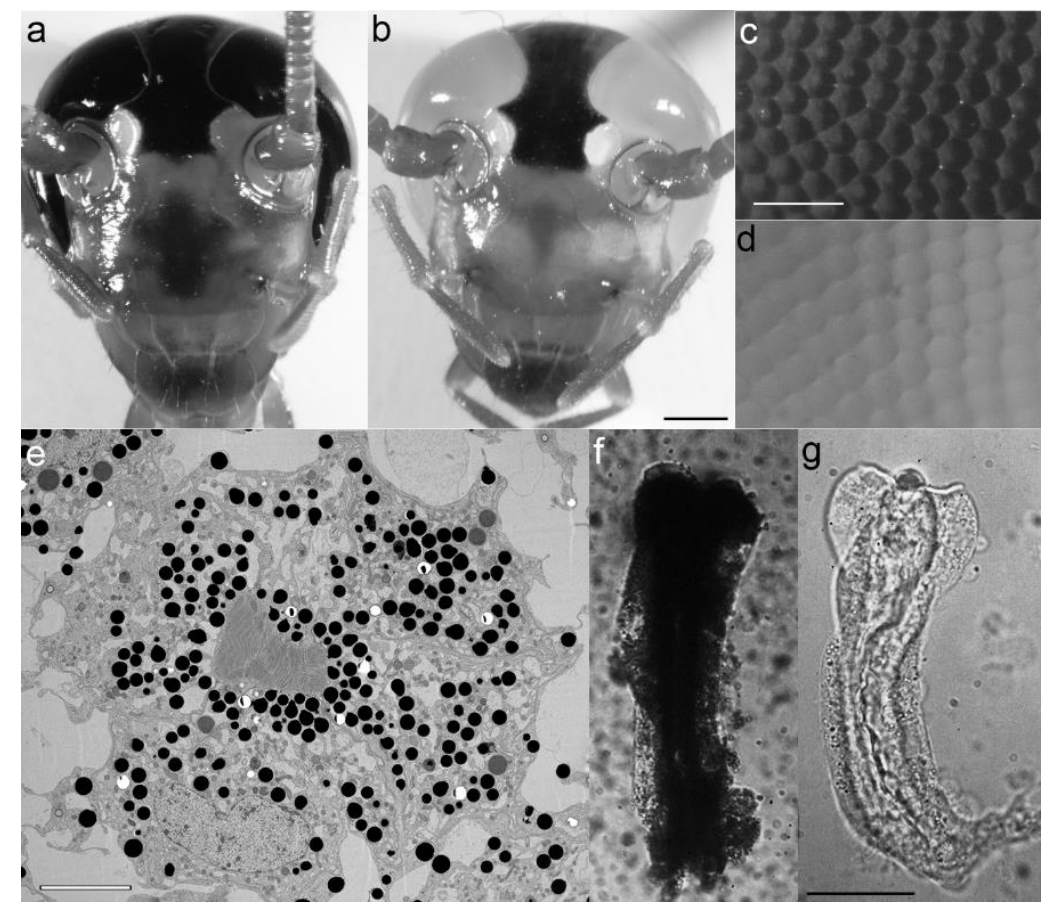

Fig. 1 Wild-type and white-eyed $P$. americana ommatidia. a, b Photographs of heads of male wild-type and W-E cockroaches, respectively; the scale bar in panel $\mathbf{b}$ is $1 \mathrm{~mm}$. c, $\mathbf{d}$ Micrographs of the corneal surfaces in the frontal part of the eye from the same animals as in $\mathbf{a}$ and $\mathbf{b}$, respectively; the scale bar in panel $\mathbf{c}$ is 100 $\mu \mathrm{m}$. e A cross-section of a wild-type ommatidium through the distal segment; the transmission electron micrograph is part of a previously published dataset (Frolov et al. 2017); the scale bar is 5 $\mu \mathrm{m}$. f, $\mathbf{g}$ Dissociated ommatidia from wild-type (f) and W-E (g) cockroaches; the scale bar is $30 \mu \mathrm{m}$.

\section{Angular sensitivity}

We hypothesized that angular sensitivity of W-E photoreceptors would be expanded due to the spread of light through the retina in the absence of screening pigment. Angular sensitivity was measured in intracellular recordings. We found that angular sensitivity in W-E animals was not only widened but consisted of two distinct components, which could best be described by superposition of two separate Gaussian functions. Figure 2 shows typical angular sensitivities of wild-type and W-E photoreceptors. In wild-type photoreceptors, the data were fitted by a single Gaussian, and the acceptance angle was determined as the half-width of the fitted function (Fig. 2a). In W-E photoreceptors, the data were fitted 
Fig. 2 Angular sensitivity. a Typical angular sensitivities of wild-type and W-E photoreceptors. Voltage response values were obtained in intracellular recordings and converted into relative sensitivities as described in Methods. Data were fitted with a single (for wild-type) or double (for WE) Gaussian functions (solid black and grey traces, respectively). b Average acceptance angles in wildtype and W-E photoreceptors; the angles were determined as half-widths of Gaussian fits in each photoreceptor. $\mathbf{c}$ The relative amplitudes of wide and narrow acceptance angle components for W-E photoreceptors; here and elsewhere $(n)$ stands for the number of cells and error bars denote standard deviation unless specified otherwise.

using a sum of two Gaussian functions, with separate acceptance angles determined for each component. The wild-type photoreceptor mean acceptance angle was $8.2 \pm 2.1$ degrees $(n=11)$, consistent with

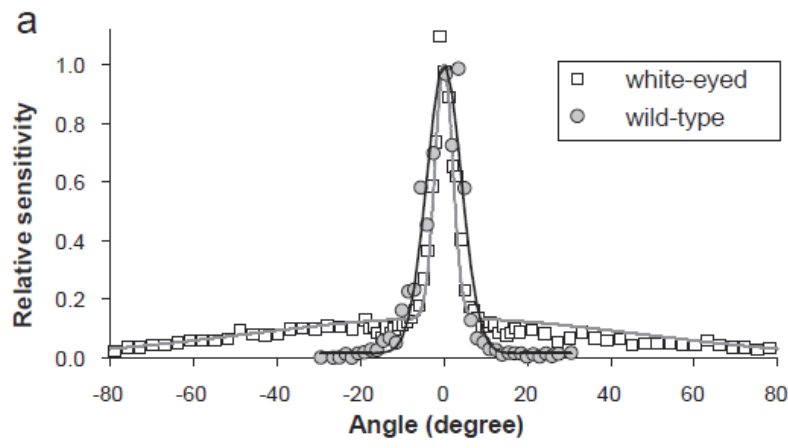
photoreceptors, the narrower component had a half-width of $7.2 \pm 2.3$ degrees, and the wider component was $122 \pm 43$ degrees ( $n=7$, Fig. 2b). The relative amplitudes of the narrow and wide components were $0.81 \pm 0.09$ and $0.19 \pm 0.08$, respectively (Fig. 2c).

\section{Membrane capacitance, absolute sensitivity and elementary responses in patch-clamp experiments}

Next, we investigated if the basic functional properties of W-E photoreceptors were different to the wildtype. We measured the resting potential, membrane capacitance $C_{\mathrm{m}}$, absolute sensitivity, and quantum bump properties. Previously, some of these parameters were shown to change when the cockroaches were reared in constant light or dark (Frolov et al. 2018). These measurements were performed using patch-clamp on dark-adapted photoreceptors. The resting potential was $54.3 \pm 5.9 \mathrm{mV}(n=19)$ in wild-type and -55.9 $\pm 5.7 \mathrm{mV}(n=7)$ in W-E photoreceptors. Mean photoreceptor $C_{\mathrm{m}}$ in W-E was much
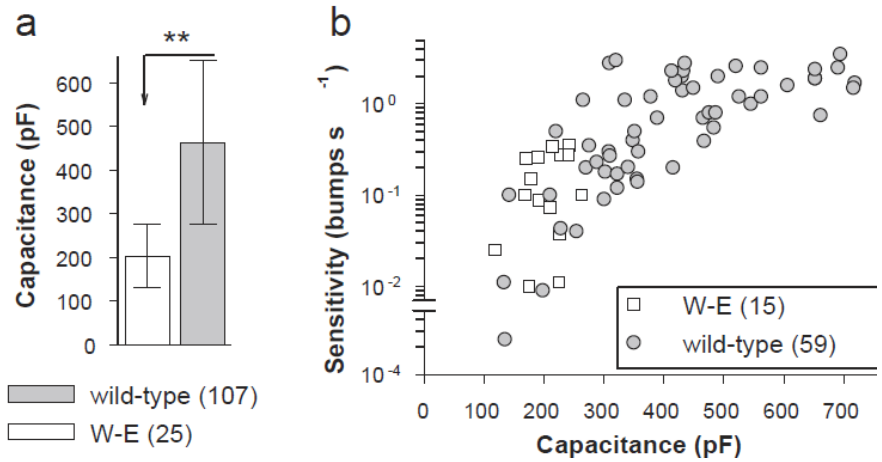

Fig. 3 Variation of photoreceptor capacitance and absolute sensitivity. a Mean capacitance values of white-eyed (W-E) and wild-type photoreceptors. Mean $C_{\mathrm{m}}$ was significantly smaller in W-E than in wildtype photoreceptors: correspondingly, $203 \pm 74(n=25)$ vs. $464 \pm 188 \mathrm{pF}\left(n=107 ; P<10^{-10}\right.$, unpaired $t$-test). b Absolute sensitivity to light was determined by counting bump rates during continuous stimulation at low light intensity eliciting < 10 bumps per second; the rates were recalculated for the common level corresponding to the $5 \cdot 10^{-6}$ light level in Fig. $5 \mathrm{c}$. The median absolute sensitivity values were $0.1(0.06: 0.27)$ in W-E $(n=15)$ and $0.75(0.19: 1.85)$ bumps $\mathrm{s}^{-1}$ in wild-type $\left(n=59 ; P<10^{-3}\right.$, MWUT) photoreceptors. Results were obtained in patch-clamp experiments. 
Fig. 4 Elementary voltage responses. a, b Voltage bumps evoked by constant light stimulation in typical W-E (a) and wild-type (b) photoreceptors during patch-clamp experiments. c Average voltage bumps. Due to strong dependence of bump amplitude on resting potential, group averages were obtained in the following way: first, mean bumps were obtained for each photoreceptor; second, a subsample of photoreceptors was selected so that their mean resting potentials were the same for the wildtype and W-E groups (see Results). Voltage bump amplitude was $1.6 \pm 1.0 \mathrm{mV}(n=15)$ in wild-type and $3.2 \pm 2.0 \mathrm{mV}(n=7 ; P=0.02$, unpaired $t$-test $)$ in W-E photoreceptors. d Normalized voltage bumps from panel c.

smaller (203 $\pm 74 \mathrm{pF} ; n=25)$ than in wild-type (464 \pm $188 \mathrm{pF} ; n=107$; Fig. 3a). Comparable decrease in $C_{m}$ was also seen when wild-type cockroaches were exposed to constant light (Frolov et al. 2018).
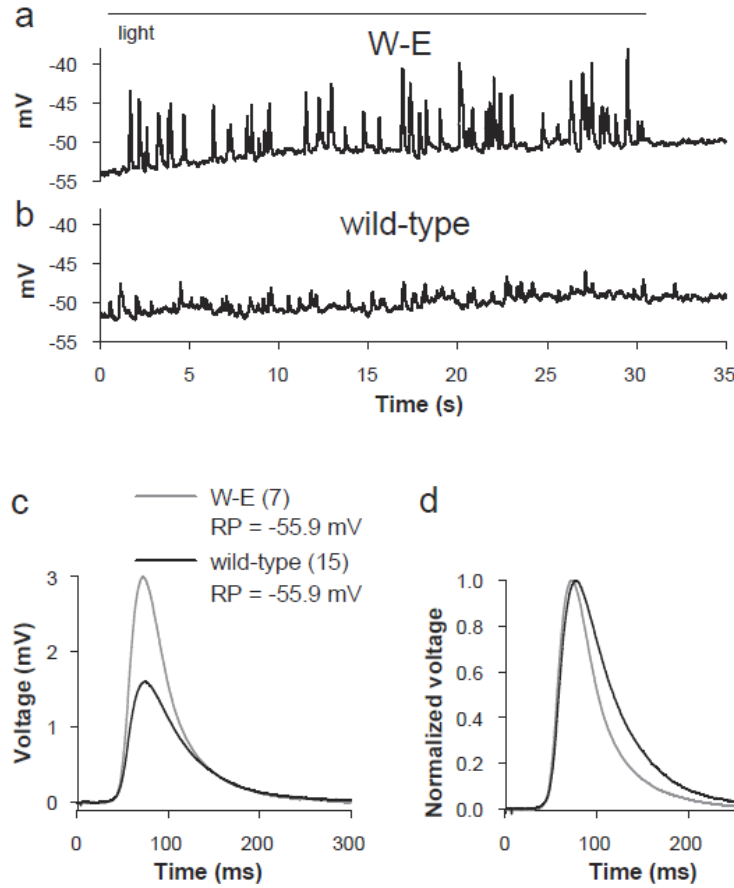

d

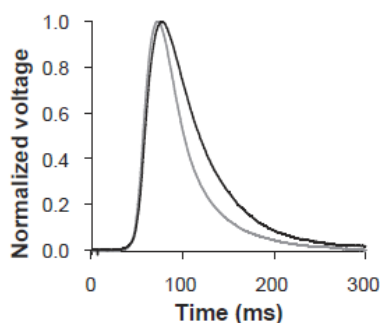

Mean bump latency was determined using flash-induced current bumps in voltage-clamp mode. The stimulus duration was $1 \mathrm{~ms}$ and its intensity was adjusted to evoke single bump responses with a probability $<0.7$. Bump latency was measured as the interval between the light flash and the bump current reaching $10 \%$ of maximum. The mean latencies for each photoreceptor were averaged to give group means. Mean latency for W-E photoreceptors was $68.9 \pm 20.5 \mathrm{~ms}(n=10)$ vs. $69.4 \pm 19.4 \mathrm{~ms}(n=49)$ for wildtype photoreceptors. Mean amplitudes of current quantum bumps were not changed in W-E in comparison to wild-type photoreceptors.

Absolute photoreceptor sensitivity was evaluated by counting current quantum bumps evoked in dim constant light. As different photoreceptors had different sensitivities, the measurements were made at different light levels, each adjusted to evoke one to ten bumps per second. These estimates were then

Fig. 5 Light-induced currents. a, b Typical lightinduced currents recorded from photoreceptors in dissociated ommatidia in W-E (a) or wild-type (b) cockroaches; 4 s light stimuli were used (horizontal lines); recordings were performed at a holding potential of $-82 \mathrm{mV}$ at five intensities in ten-fold increments (inset). c Dependence of sustained LIC on light intensity; LIC values were obtained as averages of the final three seconds of current responses; arrows are explained in Results. The differences in sustained LIC amplitudes were statistically significant even when LIC in W-E at the relative intensity 4 was compared to LIC in wild-type at intensity 0.5 (arrows): $-397 \pm 318 \mathrm{pA}$ $(n=10)$ vs. $-636 \pm 322 \mathrm{pA}(n=26 ; P=0.04$, MWUT).
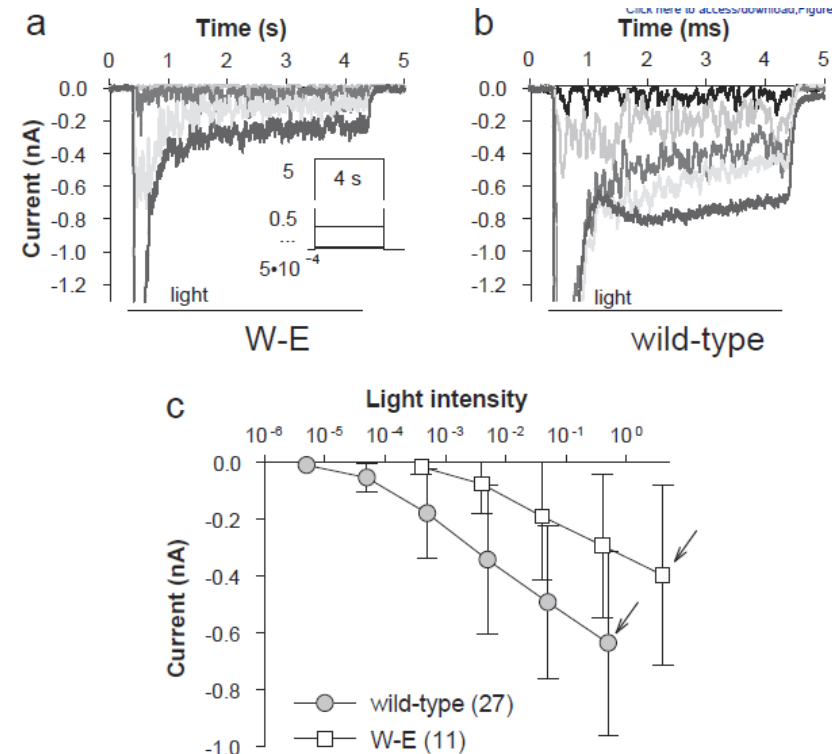
recalculated for a common light level (the lowest $5 \cdot 10^{-6}$ light level in Fig. 5c). Absolute sensitivity was significantly smaller in W-E than in wild-type photoreceptors (Fig. 3b). The reduced mean absolute sensitivity in W-E photoreceptors is consistent with the reduced mean membrane capacitance.

The combination of membrane capacitance and instantaneous membrane resistance determines the low-pass filtering of voltage signals by the photoreceptor membrane. Generally, voltage bumps evoked at resting potential by low-intensity continuous light stimulation were larger and faster in W-E than in wildtype photoreceptors (Fig. 4). To properly compare mean voltage bumps, the effect of voltage-dependent membrane conductance on input resistance needs to be controlled for by equalizing mean resting potentials in the comparison groups. We selected a subgroup of voltage bump recordings from wild-type photoreceptors with mean resting potential matching that in W-E bump recordings (Fig. 4c). The resting potential in the W-E group was $-55.9 \pm 5.7 \mathrm{mV}$, and $C_{\mathrm{m}}$ was $212 \pm 49 \mathrm{pF}(n=7)$. In the wild-type subgroup, the resting potential was $-55.9 \pm 5.6 \mathrm{mV}$, and $C_{\mathrm{m}}$ was $363 \pm 160 \mathrm{pF}(n=15)$. Mean voltage bump amplitude was significantly higher in W-E than in wild-type photoreceptors (Fig. 4c).

When the group-average bumps were normalized, a slower decay of elementary voltage responses in wild-type photoreceptors was apparent (Fig. 4d), indicating that low-pass filtering of elementary voltage signals is reduced in W-E when compared to wild-type photoreceptors due to the relatively small $C_{\mathrm{m}}$.

\section{Macroscopic light-induced current in patch-clamp experiments}

In P. americana, two light-activated channels, TRP and TRPL, contribute approximately equally to LIC (Saari et al. 2017). We have previously reported strong positive correlations between $C_{\mathrm{m}}$ and macroscopic LIC in several insect species (Frolov 2016), and found decreased LIC in constant light-exposed cockroaches (Frolov et al. 2018). Here, the relatively low $C_{\mathrm{m}}$ and absolute sensitivity of W-E photoreceptors implied that their macroscopic sustained LIC would also be smaller than in wild-type photoreceptors. Representative recordings of LIC from a W-E and wild-type photoreceptors elicited by $4 \mathrm{~s}$ constant light pulses in 10-fold increments are shown in Fig. 5a and b. LIC amplitudes in W-E photoreceptors were significantly smaller on average than in wild-type (Fig. 5c).

\section{Sustained potassium current in patch-clamp experiments}

P. americana photoreceptors have several voltage-gated $\mathrm{K}^{+}(\mathrm{Kv})$ conductances. EAG channels give a sustained $\mathrm{Kv}$ conductance, which is responsible for setting the resting potential, countering depolarization by LIC and determining the membrane time constant (Immonen et al. 2017). If the size of the soma of the W-E photoreceptor would be different to that of the wild type, the amplitudes of $\mathrm{Kv}$ currents would also be different. However, we did not find any such differences between W-E and wild-type photoreceptors. Sustained conductance-voltage relationships were obtained from current-voltage relationships as described previously (Frolov et al. 2018). The plots were fitted with a first-order sigmoidal function. Mean maximal conductance was $37.3 \pm 13.0 \mathrm{nS}$ in wild-type $(n=40)$ and $37.5 \pm 18.9$ in W-E photoreceptors $(n=17)$. Mean half-activation potential values were $15.0 \pm 3.0$ and $14.3 \pm 2.0 \mathrm{mV}$ in wild-type and W-E photoreceptors, respectively. Mean slope factors were $-25.6 \pm 7.7$ and $-23.8 \pm 7.5 \mathrm{mV}^{-1}$ in wild-type and 
W-E photoreceptors, respectively. These results are consistent with the hypotheses that: (1) Kv channels are expressed in the soma that is similar in size in W-E and wild-type photoreceptors, and (2) that the differences in $C_{\mathrm{m}}$ between W-E and wild-type photoreceptors are mainly caused by differences in the size of the rhabdomere.

\section{Signal processing in intracellular recordings}

To investigate potential changes in information processing in W-E photoreceptors, we used a $60 \mathrm{~s}$ GWN light stimulus (see Methods) in intracellular recordings, at several light levels in 10-fold intensity increments. The voltage responses of W-E photoreceptors were generally similar to the responses of wildtype ones (Fig. 6; see typical responses of wild-type photoreceptors to the same GWN in the previous study (Saari et al. 2017)). $20 \mathrm{~s}$ segments of recordings at each light level are shown in Fig. 6a. The corresponding signal gain, signal power, noise power and SNR functions are shown in Figs. 6b-e, respectively. It can be seen that as signal amplitude increased with light level, noise decreased. As a result, SNR and information rates also increased (Fig. 6e, f).
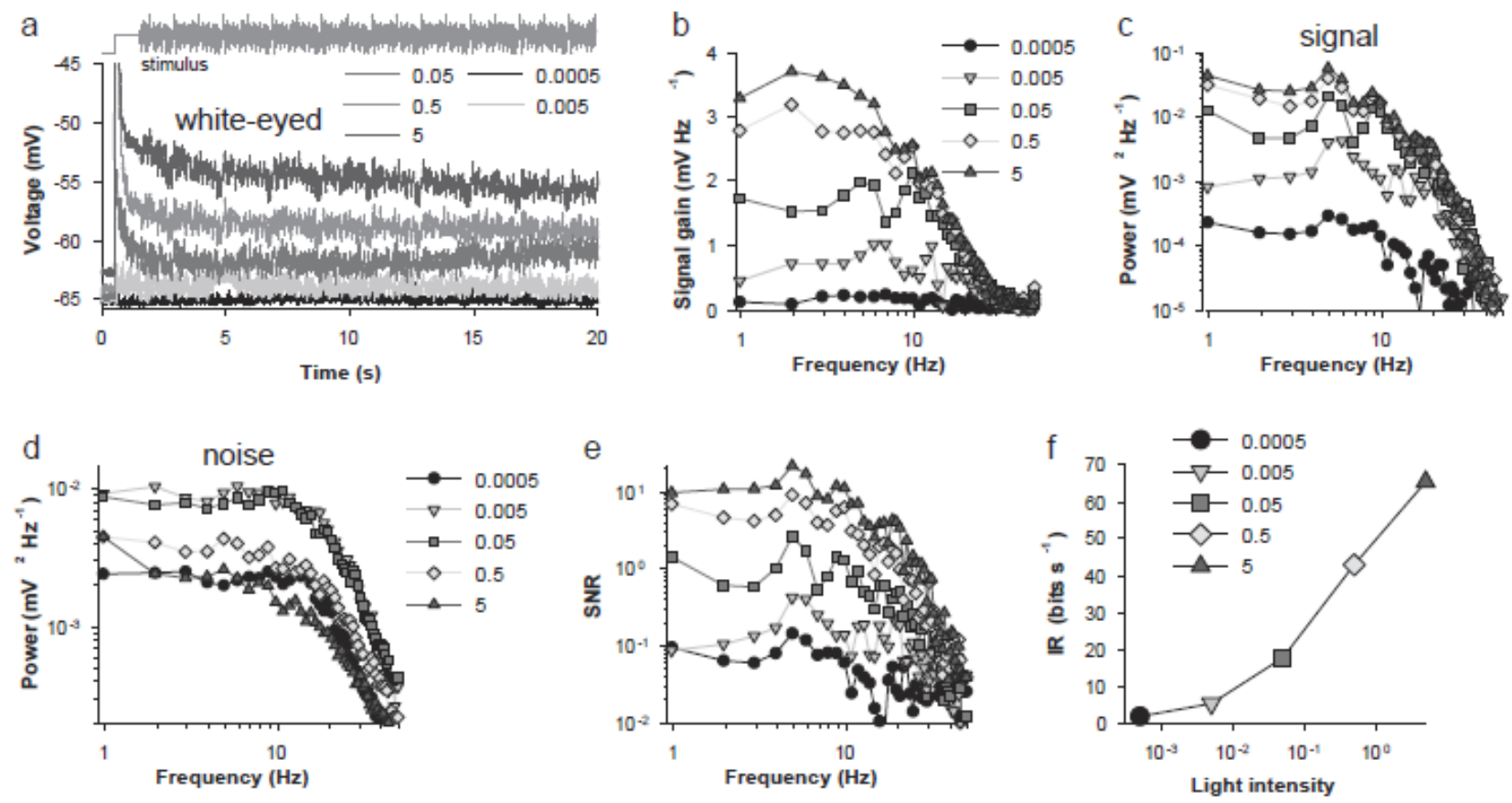

Fig. 6 Intracellular responses of white-eyed photoreceptors to GWN stimulation. a First $20 \mathrm{~s}$ of representative voltage responses of a W-E photoreceptor to a $60 \mathrm{~s} \mathrm{GWN} \mathrm{stimulus} \mathrm{at} \mathrm{five} \mathrm{light} \mathrm{levels;} \mathrm{the}$ GWN stimulus is shown above. b-e Dependencies of signal gain (b), signal power (c), noise power (d), and SNR (e) on frequency for the same photoreceptor; color coding is consistent for all panels; legends in panels $\mathbf{a}, \mathbf{b}, \mathbf{d}$, and $\mathbf{f}$ denote the light levels as explained in Methods. $\mathbf{f}$ Dependence of information rate on light level for the same cell.

The average parameters associated with information processing at different light intensities are compared in Fig. 7. Sustained membrane depolarization during light response was essentially the same in W-E and wild-type photoreceptors (Fig. 7a). Information rates averaged at each light level are plotted in Fig. 7b. At the highest light level, mean IR in W-E photoreceptors significantly exceeded mean IR of wildtype photoreceptors. However, mean $\mathrm{IR}_{\max }$ values were not significantly different (Fig. 7c). 

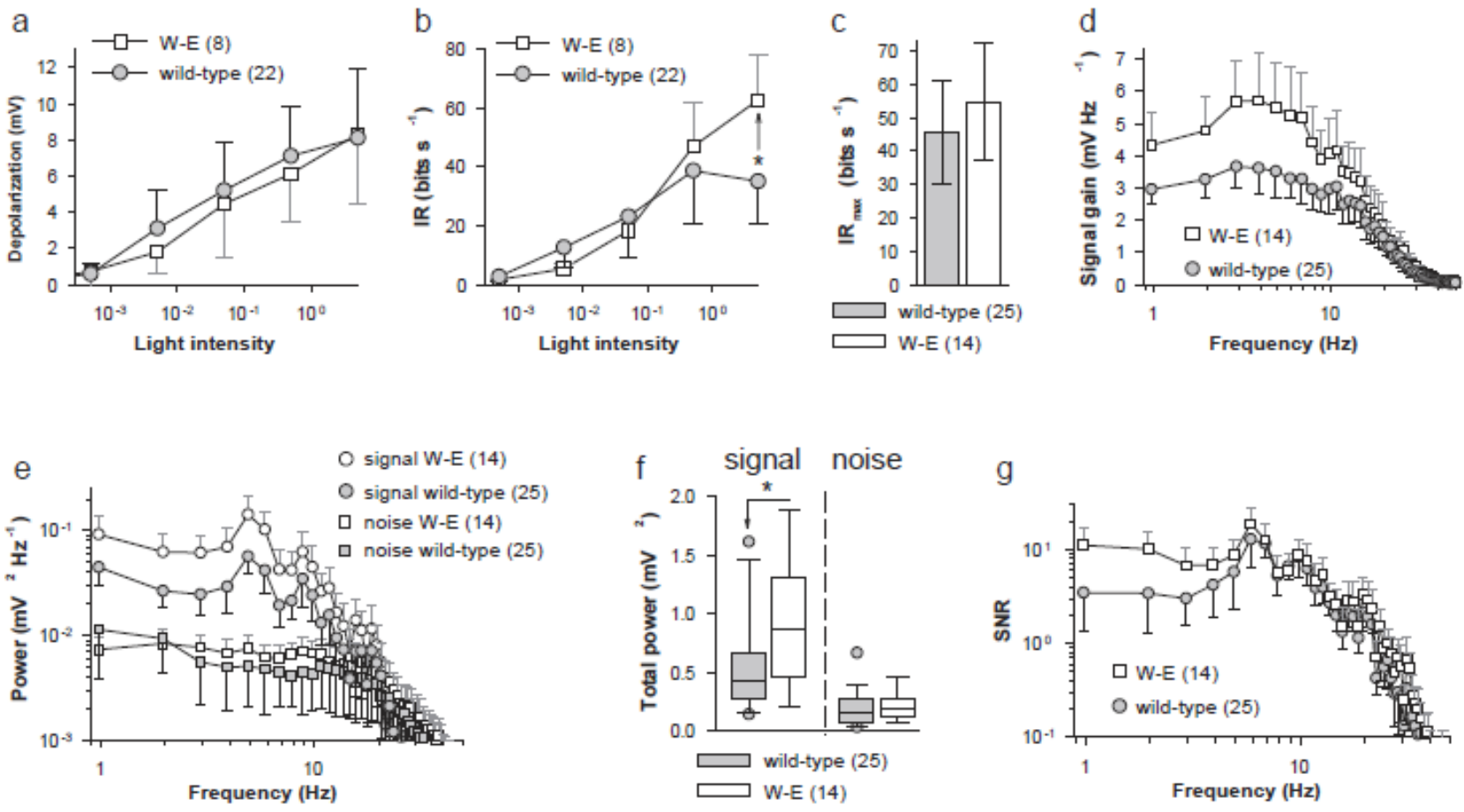

Fig. 7 Comparison of information processing in W-E and wild-type photoreceptors by intracellular recording. a Dependencies of average sustained membrane depolarization on light level during responses to GWN are shown for W-E and wild-type photoreceptors; values were obtained by averaging plateau voltage during the entire response except the first second and then subtracting the resting potential. b Dependencies of mean information rates on light level; only a subset of photoreceptors with responses obtained at the same light levels was used; at the light level of 5, IR was $35 \pm 15$ in wild-type bits $\mathrm{s}^{-1}$ in control $(n=12)$ and $63 \pm 16$ bits s$^{-1}$ in W-E photoreceptors $\left(n=5 ; P=0.003\right.$, unpaired $t$-test). $\mathbf{c}$ Mean $\mathrm{IR}_{\max }$ values were not significantly different between the groups: $54.7 \pm 17.7$ bits s$^{-1}$ in W-E $(n=14)$ vs. $45.8 \pm$ 15.5 bits s$^{-1}$ in wild-type photoreceptors $(n=25 ; P=0.12$, unpaired $t$-test). d Median signal gain functions for responses to GWN at light intensities that elicited $\mathrm{IR}_{\max }$ responses; when signal gain functions were integrated over the range from 1 to $50 \mathrm{~Hz}$, the total gain was $0.93 \pm 0.31 \mathrm{~V}$ in W-E $(n=14)$ and $0.64 \pm$ $0.24 \mathrm{~V}$ in wild-type photoreceptors $(n=25 ; P=0.007$, unpaired $t$-test), error bars here and in panels $\mathbf{e}$ and g are m.a.d. e Median signal and noise power spectra in W-E and wild-type photoreceptors; data were obtained from $\mathrm{IR}_{\max }$ responses. f Box plots compare total signal and noise power spectra integrals in 1 - 50 $\mathrm{Hz}$ range for $\mathrm{IR}_{\max }$ responses; error bars denote the interquartile range; circles denote $5^{\text {th }} / 95^{\text {th }}$ percentile outliers. The total signal power was $0.87(0.53: 1.20) \mathrm{mV}^{2}$ in W-E $(n=14)$ and $0.43(0.29: 0.65) \mathrm{mV}^{2}$ in wild-type photoreceptors $(n=25 ; P=0.02$, MWUT). $g$ Median SNR in W-E and wild-type photoreceptors; data were obtained from $\mathrm{IR}_{\max }$ responses.

Next, we assessed recordings associated with the maximal information rate $\left(\mathrm{IR}_{\max }\right)$ in each photoreceptor (Fig. 7d-g). The signal gain was higher in W-E than in wild-type (Fig. 7d). There was no difference in mean membrane corner frequency values obtained from $\mathrm{IR}_{\max }$ responses (data not shown). Consistent with the increased signal gain, median signal power was higher in W-E than in wild-type photoreceptors, whereas median noise power was not statistically different (Fig. 7e, f). It can be seen that the differences in signal power are mainly concentrated in the low-frequency region. Accordingly, the median SNR function for W-E was higher than that for wild-type in the low-frequency region (Fig. $7 \mathrm{~g}$ ).

\section{Opsin gene expression}

We measured the expression levels of mRNA for the three opsin genes that were previously identified in the cockroach retina (French et al. 2015). In the wild-type retina, one green-sensitive opsin (GO1) is 
expressed at a much higher level than the UV opsin (UVO), while the second green-sensitive opsin (GO2) is expressed at a significantly lower level than either of the other two opsins (French et al. 2015). Here, we hypothesized that increased exposure to light in W-E retina could result in lower expression levels of these opsins. Our results show that both GO1 and UVO were downregulated in the W-E retina when compared to wild-type. However, an unexpected finding was that the green opsin, GO2, which is expressed at a low level in the wild-type retina, was dramatically upregulated (37-fold) in the W-E retina (Fig. 8).

\section{Behavioural differences}

We hypothesized that differences in absolute sensitivity between W-E and wild-type cockroaches could lead to differences in behaviour. In a previous study we showed that locomotor and other behaviours of wild-type cockroaches change with the level of illumination (Zhukovskaya et al. 2017). We compared locomotor behaviours of W-E and wild-type cockroaches using the same experimental and analytical methodology. Naive cockroaches voluntarily walked into a plain testing chamber illuminated with dim red light. Video recordings of exploratory activity were performed with an infrared camera for $30 \mathrm{~min}$ in dim red light ("session 1" or S1). 10 min after the end of the first recording, the chamber was lit with either dim red or dim green light, and a further $30 \mathrm{~min}$ of cockroach behaviour was recorded ("session 2" or S2).

We found that locomotor activity of W-E cockroaches during S1 was similar to wild-type

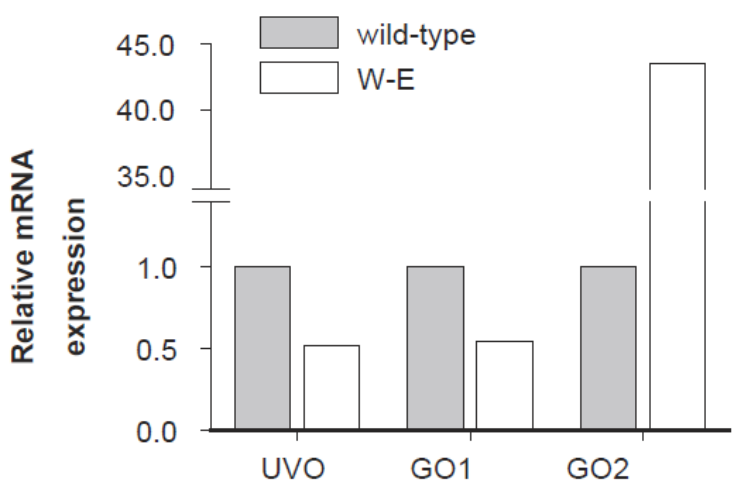

Fig. 8 Quantitative PCR for opsin levels. Relative expression of $\mathrm{UVO}, \mathrm{GO} 1$ and $\mathrm{GO} 2$ genes in W-E and wild-type photoreceptors. Reference genes were actin and GAPDH and the data were normalized to wild-type mRNA expression. The results represent the means of three repetitions of mRNA measurement using samples of 14 wild-type and $16 \mathrm{~W}$-E retinas as described in Methods; W-E values were divided by the respective wild-type values; s.e.m. of the technical replication did not exceed 9\%; the break in ordinate scale accommodates $\mathrm{GO} 2$ expression level in W-E. cockroaches (Fig. 9a). While average values indicated that W-E were more agitated than the wild-type cockroaches, these differences were due to strongly agitated behaviour of only 3 of 23 animals. During S2 in dim red light the exploratory activity of both groups decreased equally (Fig. 9b). However, differences were observed in dim green light, which caused a strong increase in locomotor activity compared to dim red in wild-type cockroaches, as shown previously (Zhukovskaya et al. 2017). However, W-E activity during S2 in dim green light was not different from control (S1) and they were significantly less active than wild-type cockroaches under the same conditions (Fig. 9b). Other behaviours, such as grooming, under dim green light were similar to dim red S2 controls (data not shown). These results are consistent with the decreased sensitivity to light of W-E cockroaches.

\section{Discussion}


In this work we compared the functions of a white-eyed mutant $P$. americana photoreceptors to those of wild-type using in vivo intracellular recordings, in vitro patchclamp experiments, quantitative measurement of opsin gene expression, and a behavioural assay. Naturally-occurring white-eyed mutants have been isolated from wild populations at least twice (Ross et al. 1964). The first observation was made by Jefferson (Jefferson 1958) who found W-E cockroaches in a deep coal mine in Glamorgan, Wales. The strain used in the current study, pearl, was later isolated by Ross et al. (Ross et al. 1964). While these two strains may represent the same recessive mutation, this has not been established because complementation test has not been performed. It should also be noted that the changes in electrophysiological properties of W-E mutants observed in this study and interpreted as plasticity (see below) did not necessarily occur in their original coal mine habitat.

The changes in photoreceptor function in white-eyed mutants could arise from three

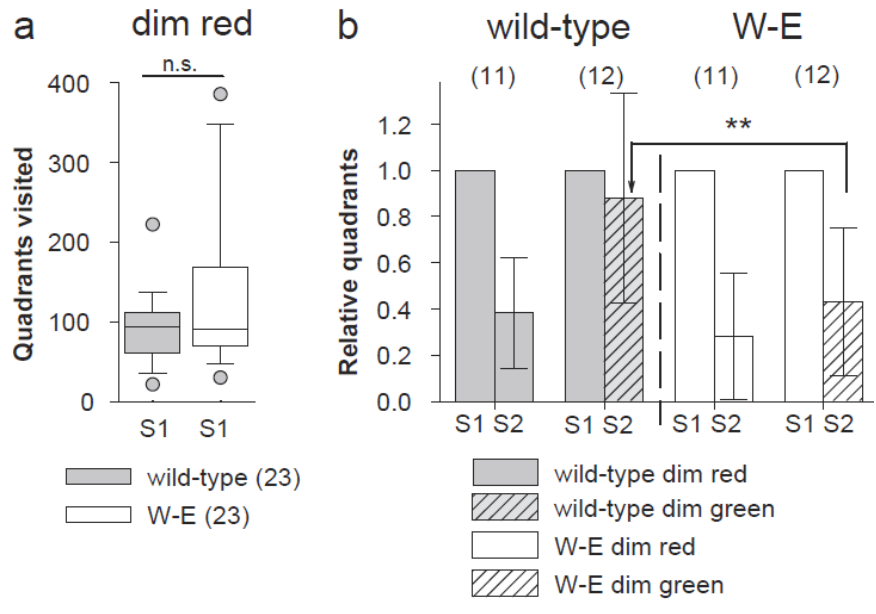

Fig. 9 Locomotor behaviour. a Locomotor activity in dim red light during session 1 (S1) of exploring the testing chamber did not differ significantly between wild-type and W-E cockroaches $(P=0.19$, MWUT); circles denote $5^{\text {th }} / 95^{\text {th }}$ percentile outliers; n.s. indicates absence of statistically significant differences. b Relative locomotor activity in dim red light (during both S1 and S2) or dim green light (dim red light during S1 and dim green light during S2) exposure experiments; values were obtained by dividing the number of quadrants visited during S2 by the number of quadrants visited during S1 for each animal; numbers in parentheses denote the number of experiments. Locomotor activity during S2 in green light exposure experiments decreased significantly in W-E in comparison to wild-type cockroaches $(P=0.006$, MWUT). (see Fig. 1 in (Zhukovskaya et al. 2017) for description of the experimental setup and procedure). possible sources: removal of the actual screening functions, phenotypic plasticity, and unrelated effects of the mutated gene. Our findings can be explained by the first two categories. Absence of the screening pigment altered the angular sensitivity of the dark-adapted W-E photoreceptors. This was an expected consequence of the loss of screening pigment and cannot be considered an adaptive or compensatory adjustment. The changes in $C_{\mathrm{m}}$, absolute sensitivity, LIC amplitudes, voltage bumps, and UVO and GO1 concentrations together with the absence of any changes in phototransduction, are important since they outline the electrophysiological phenotype of W-E photoreceptors and allow us to understand the mechanism of the putative compensatory changes. These results have similarities with recent findings in wild-type cockroaches exposed to constant light for several months (Frolov et al. 2018) and are discussed below.

It is not known if the W-E mutation is associated with other molecular changes than the lack of screening pigment that could affect the photoreceptor function. In insects, brown screening pigment is usually a derivative of tryptophan, and tryptophan levels might be increased in the pearl strains (Ward and 
Hammen 1957; Ross et al. 1964). However, it is not known if elevated tryptophan can affect photoreceptor function.

\section{Plasticity-related changes}

Photoreceptor function is largely determined by two groups of factors. Those dependent on the area of the light-sensitive rhabdomeric or light-insensitive somatic membrane can be defined as extensive or quantitative parameters as they scale up or down with the size of the appropriate part of the cell. Examples include absolute $C_{\mathrm{m}}$, sensitivity to light, amplitudes of LIC and Kv currents. Other factors, which do not scale up with the size of the photoreceptor, can be defined as intensive ones. These include the amplitudes of quantum bumps, their latency, and the slope factor of the potassium current, Kv (Frolov et al. 2018). Previous studies have found that variability in the photoreceptor membrane area in P. americana and several other species, as estimated by membrane capacitance, is strongly correlated with variations in absolute sensitivity, the amplitude of macroscopic LIC, and some other parameters (Frolov 2016). On the other hand, no link has been found between those parameters and the properties of current quantum bumps or phototransduction latency in dark-adapted photoreceptors.

Results of our recent research into the effects of prolonged exposure to constant light or dark in wild-type cockroach photoreceptors (Frolov et al. 2018) are summarized in Table 1 and compared to the results of the present study. Values for four basic electrophysiological properties of W-E photoreceptors are between values measured after constant light exposure and wild-type/control photoreceptors, suggesting that the periodic exposure of the W-E cockroaches to light may have somewhat similar effect than exposure of wild-type cockroaches to constant light. The main changes in electrophysiological properties after prolonged light or dark exposure were suggested to be caused by the structural remodeling of the rhabdomere (Frolov et al. 2018). The results reported here also indirectly indicate that the differences in photoreceptor function between the W-E and wild-type strains may result from differences in the size of the rhabdomere as estimated by membrane capacitance.

Four of our findings from W-E photoreceptors suggest that morphological changes are restricted to the rhabdomere: $\mathrm{Kv}$ conductance was unchanged; absolute sensitivity and LIC were lower; and voltage bumps were larger than in the wild-type photoreceptors. In D. melanogaster the Kv channels are located in the soma, not in the rhabdomere (Hardie and Raghu 2001). If these channels are also in the soma of cockroach photoreceptors and their density is similar to Drosophila, our results suggest that the size of the soma is approximately the same in W-E and wild-type photoreceptors. The relatively low LIC points to modifications in the rhabdomere. A decrease in LIC could be caused by a decrease in LIC density (current divided by capacitance) due to changes in expression of light-activated channels, in the number or size of the microvilli. The differences in $C_{\mathrm{m}}$ and absolute sensitivity indicate that the latter scenario is most plausible (Frolov et al. 2018). The lower levels of the dominant green opsin GO1 and UVO genes are also consistent with this conclusion. However, since mRNA levels do not correspond directly to protein levels, these results must be interpreted with caution. 
Significantly higher expression of the secondary green opsin GO2 in W-E mutants is an interesting finding (Fig. 8). In wild-type cockroach retina, GO1 is approximately 100 times more abundant than GO2 (French et al., 2015). GO1 is orthologous to the cricket (Gryllus bimaculatus) Green B opsin that is expressed in compound eyes while the $\mathrm{GO} 2$ is orthologous to the cricket Green A opsin that was found in ocelli (Henze et al 2012). The cricket green, blue and UV opsins are located in different areas of the compound eye giving different spectral sensitivities to central and peripheral regions of the eye (Henze et al 2012). The cockroach compound eye does not have blue opsin (French et al., 2015) but the two green opsins and UVO may be expressed in different regions of the eye. In the W-E mutant, rhabdomere size may be specifically smaller in those areas expressing GO1 and UVO, compared to a relatively larger area containing GO2. Another possibility is that the excessive amount of light entering the retina triggers high GO2 expression because the diminished rhabdomere cannot produce adequate amounts of GO1. In the wildtype cockroaches exposed to constant light only a small elevation of GO2 was observed (Frolov et al. 2018) suggesting that different mechanisms are involved in the mutant eye and the light exposed normal eye.

The high-amplitude, fast voltage bumps found in W-E photoreceptors exemplify the functional consequences of the smaller rhabdomere size. Low-pass filtering by the membrane depends on the membrane time constant $\tau$ as $f_{3 \mathrm{~dB}}=1 /(2 \pi \cdot \tau)$, where $f_{3 \mathrm{~dB}}$ is the membrane corner frequency. In turn, $\tau=$ $R_{\mathrm{m}} \cdot C_{\mathrm{m}}$, where $R_{\mathrm{m}}$ is the instantaneous membrane resistance, or, in the dark-adapted photoreceptor, the input resistance. If the reduction in rhabdomere size were accompanied by a proportional decrease in the area of light-insensitive membrane, input resistance would be expected to increase as it is mainly determined by the sustained Kv conductance (Immonen et al. 2017). Such an increase would offset the relaxation of lowpass filtering due to decreased $C_{\mathrm{m}}$, and prevent increase in voltage bump amplitudes, which would be inconsistent with our observations.

In intracellular recordings, W-E photoreceptors responded to GWN with a slightly higher mean $I R_{\max }$ than the wild-type ones, due to higher signal gain and power. Moreover, at the brightest light level mean IR was significantly higher in W-E than in wild-type photoreceptors. These results were generally consistent with the findings in flies (Howard et al. 1987). However, despite the relatively small $C_{\mathrm{m}}$, no increase in membrane corner frequency was observed, indicating that the available bandwidth provided by $\mathrm{Kv}$ conductances is sufficient for lossless accommodation of light responses generated by LIC both in wildtype and W-E photoreceptors in accordance with the matched filtering concept (Laughlin 1996). Increased signal gain in W-E photoreceptors is probably caused by improved effective illumination of the photoreceptor by stray light, which under these experimental conditions does not add noise. However, visual stimulation arriving from an actual natural scene, while changing from point to point in space, is partly spatially correlated (Ignatova et al. 2018). Thus, stray light produced by summation across the retina would not be constant but partly contrast-modulated and probably out of phase with the light propagating along the optical axis of the ommatidium. This would be expected to add noise to the system and decrease its information capacity.

\section{Light sensitivity}


Is visual acuity strongly disrupted by the absence of screening pigment? The changes observed in angular sensitivity of W-E photoreceptors might not be expected to have a major effect because it was still dominated by the normal narrow component, while the average relative amplitude of the wide component did not exceed $20 \%$. This suggests that visual acuity could be close to wild type in W-E compound eyes. However, other findings contradict this hypothesis. Visual acuity is based on the ability of photoreceptors and downstream neural centers to reliably discriminate between visual signals arriving from neighboring points in space. In the normal eye, visual acuity primarily depends on the interommatidial and acceptance angles. In the absence of the screening pigment, the stray light might contribute to excessive excitation of photoreceptors to a much greater degree than would be expected from the angular sensitivity. Since the ambient light illuminates the entire retina, the relative amplitude of the wide component will probably be higher and degrade spatial resolution more than under the conditions of angular sensitivity measurements. However, under normal nocturnal illumination conditions that favor sensitivity over acuity, such a loss may not be functionally important.

W-E cockroaches did not demonstrate any changes in exploratory behavior in dim red light, which is barely visible to human eye and practically invisible to cockroaches (Zhukovskaya et al. 2017). However, there were significant changes under green light, suggesting that $\mathrm{W}$-E cockroaches are overall less sensitive to light than their wild-type counterparts. This is fully consistent with the relatively low absolute sensitivity of their photoreceptors found in our electrophysiological experiments.

Another important caveat follows from the comparison of photographs of wild-type and mutant ommatidia (Fig. 1f, g): during side-on stimulation in patch-clamp experiments, a substantial fraction of light is likely to be absorbed by the dense pigment sheath before reaching the rhabdomeres of wild-type photoreceptors. The fact that the absolute sensitivities of W-E photoreceptors did not stand out from the scatter plot cloud of wild-type data points (Fig. 3b) indicates that wild-type photoreceptors are inherently more sensitive to light than W-E photoreceptors that have the same average $C_{\mathrm{m}}$. This finding is consistent with the decrease in GO1 mRNA in W-E compared to wild-type retina (Fig. 8).

\section{Conclusions}

We have reported a suite of changes in $P$. americana photoreceptors associated to the loss of screening pigment. These changes can be categorized into two groups: those directly related to the loss of function of the screening pigment, and the accompanying compensatory or plasticity-related changes. Many of our findings from white-eyed mutants were analogous to those from wild-type photoreceptors exposed to constant light (Frolov et al. 2018) and suggest that both conditions lead to reduced rhabdomere size.

\section{Competing interests}

No competing interests declared. 
Table 1. Comparison of electrophysiological properties of wild-type photoreceptors exposed to different light regimens and in W-E photoreceptors.

\begin{tabular}{|c|c|c|c|c|}
\hline & $\begin{array}{c}\text { Constant light } \\
24 \mathrm{~h} \mathrm{~L}\end{array}$ & $\begin{array}{c}\text { White-eyed } \\
12 \text { h L: } 12 \text { h D }\end{array}$ & $\begin{array}{c}\text { Wild-type/control } \\
12 \text { h L: } 12 \text { h D }\end{array}$ & $\begin{array}{c}\text { Constant dark } \\
24 \mathrm{~h} \mathrm{D}\end{array}$ \\
\hline$C_{\mathrm{m}}, \mathrm{pF}$ & $157 \pm 58(21)$ & $203 \pm 74(25)$ & $464 \pm 188(107)$ & $560 \pm 149(26)$ \\
\hline Median AS, bumps s ${ }^{-1}$ at LI of $5 \cdot 10^{-6}$ & $1.2 \cdot 10^{-3}(15)$ & $0.1(15)$ & $0.75(59)$ & $5.3(9)$ \\
\hline Median LIC at LI of $5 \cdot 10^{-1}, \mathrm{pA}$ & $-165(8)$ & $-345(10)$ & $-636(26)$ & $-887(8)$ \\
\hline Bump latency, ms & $54.4 \pm 7.8(12)$ & $68.9 \pm 20.5(10)$ & $69.4 \pm 19.4(49)$ & $66.1 \pm 9.2(8)$ \\
\hline
\end{tabular}

Data for photoreceptors exposed to constant light or dark were published previously (Frolov et al. 2018).

Wild-type and white-eyed data are from the present study. Abbreviations: AS, absolute sensitivity; L, light; $\mathrm{D}$, dark; $C_{\mathrm{m}}$, membrane capacitance; LIC, light induced current. LI, light intensity. Values are expressed as means \pm SD for normally distributed and as medians for non-normally distributed dat. Numbers of experiments are given in parentheses.

\section{References}

Alawi AA, Jennings V, Grossfield J, Pak WL (1972) Phototransduction mutants of Drosophila melanogaster. In: Arden GB (ed) The visual system: Neurophysiology, biophysics, and their clinical applications. Springer US, Boston, MA, pp 1-21.. https://doi.org/10.1007/978-1-46848231-7_1

Arikawa K, Mizuno S, Scholten DGW, Kinoshita M, Seki T, Kitamoto J, Stavenga DG (1999) An ultraviolet absorbing pigment causes a narrow-band violet receptor and a single-peaked green receptor in the eye of the butterfly Papilio. Vision Res 39:1-8. https://doi.org/https://doi.org/10.1016/S0042-6989(98)00070-4

Arikawa K, Pirih P, Stavenga DG (2009) Rhabdom constriction enhances filtering by the red screening pigment in the eye of the Eastern Pale Clouded yellow butterfly, Colias erate (Pieridae). J Exp Biol 212:2057-2064. https://doi.org/10.1242/jeb.030692

Borycz J, Borycz JA, Kubow A, Lloyd V, Meinertzhagen IA (2008) Drosophila ABC transporter mutants white, brown and scarlet have altered contents and distribution of biogenic amines in the brain. $\mathrm{J}$ Exp Biol 211:3454-3466. https://doi.org/10.1242/jeb.021162

Butler R, Horridge GA (1973) The electrophysiology of the retina of Periplaneta americana L. J Comp Physiol 83:279-288. https://doi.org/10.1007/bf00693679

French AS, Meisner S, Liu H, Weckstrom M, Torkkeli PH (2015) Transcriptome analysis and RNA interference of cockroach phototransduction indicate three opsins and suggest a major role for TRPL channels. Front Physiol 6:207. https://doi.org/10.3389/fphys.2015.00207

Frolov RV (2016) Current advances in invertebrate vision: insights from patch-clamp studies of photoreceptors in apposition eyes. J Neurophysiol 116:709-723. https://doi.org/10.1152/jn.00288.2016

Frolov RV, Immonen EV, Saari P, Torkkeli PH, Liu H, French AS (2018) Phenotypic plasticity in Periplaneta americana photoreceptors. J Gen Physiol. https://doi.org/10.1085/jgp.201812107

Frolov RV, Matsushita A, Arikawa K (2017) Not flying blind: a comparative study of photoreceptor function in flying and non-flying cockroaches. J Exp Biol 220:2335-2344. https://doi.org/10.1242/jeb.159103

Gribakin FG (1988) Photoreceptor optics of the honeybee and its eye colour mutants: the effect of screening pigments on the long-wave subsystem of colour vision. J Comp Physiol A 164:123140. https://doi.org/10.1007/bf00612726

Gribakin FG, Alekseyev YN, Ukhanov KY (1996) Spectral sensitivity of white-eyed insect mutants in the UV, blue and green. J Photochem Photobiol B 35:13-18. https://doi.org/https://doi.org/10.1016/1011-1344(96)07313-7

Hardie RC, Raghu P (2001) Visual transduction in Drosophila. Nature 413:186-193. https://doi.org/10.1038/3509300235093002 [pii] 
Heimonen K, Salmela I, Kontiokari P, Weckstrom M (2006) Large functional variability in cockroach photoreceptors: optimization to low light levels. J Neurosci 26:13454-13462. https://doi.org/26/52/13454 [pii]10.1523/JNEUROSCI.3767-06.2006

Howard J, Blakeslee B, Laughlin SB (1987) The intracellular pupil mechanism and photoreceptor signal: noise ratios in the fly Lucilia cuprina. Proc Biol Sci 231:415-435. https://doi.org/10.1098/rspb.1987.0053

Ignatova, II, French AS, Frolov RV (2018) Effects of phase correlations in naturalistic stimuli on quantitative information coding by fly photoreceptors. J Neurophysiol. https://doi.org/10.1152/jn.00017.2018

Immonen EV, French AS, Torkkeli PH, Liu H, Vahasoyrinki M, Frolov RV (2017) EAG channels expressed in microvillar photoreceptors are unsuited to diurnal vision. J Physiol. https://doi.org/10.1113/JP273612

Immonen EV, Ignatova I, Gislen A, Warrant E, Vahasoyrinki M, Weckstrom M, Frolov R (2014) Large variation among photoreceptors as the basis of visual flexibility in the common backswimmer. Proc Biol Sci 281. https://doi.org/10.1098/rspb.2014.117720141177 [pii]

Jefferson GT (1958) A white-eyed mutant form of the American cockroach, Periplaneta americana (L.). Nature 182:892. https://doi.org/10.1038/182892b0

Kalmus H (1943) The optomotor responses of some eye mutants of Drosophila. Journal of Genetics 45:206-213. https://doi.org/10.1007/bf02982936

Kirschfeld K, Franceschini N (1969) Ein Mechanismus zur Steuerung des Lichtflusses in den Rhabdomeren des Komplexauges von Musca. Kybernetik 6:13-22. https://doi.org/10.1007/bf00288624

Lall AB, Strother GK, Cronin TW, Seliger HH (1988) Modification of spectral sensitivities by screening pigments in the compound eyes of twilight-active fireflies (Coleoptera: Lampyridae). J Comp Physiol A 162:23-33. https://doi.org/10.1007/bf01342700

Laughlin SB (1996) Matched filtering by a photoreceptor membrane. Vision Res 36:1529-1541

Mote MI, Goldsmith TH (1970) Spectral sensitivities of color receptors in the compound eye of the cockroach Periplaneta. J Exp Zool 173:137-145. https://doi.org/10.1002/jez.1401730203

Pak WL, Lidington KJ (1974) Fast electrical potential from a long-lived, long-wavelength photoproduct of fly visual pigment. J Gen Physiol 63:740-756

Ross MH, G. Cochran D, Smyth T (1964) Eye-color mutations in the American cockroach, Periplaneta americana. Ann Entomol Soc Am 57:790-792. https://doi.org/10.1093/aesa/57.6.790

Saari P, French AS, Torkkeli PH, Liu H, Immonen EV, Frolov RV (2017) Distinct roles of light-activated channels TRP and TRPL in photoreceptors of Periplaneta americana. J Gen Physiol 149:455464. https://doi.org/10.1085/jgp.201611737

Shannon CE (1949) Communication in the presence of noise. Proceedings of the IRE 37:10-21. https://doi.org/10.1109/JRPROC.1949.232969

Stavenga DG (1989) Pigments in compound eyes. In: Stavenga DG, Hardie RC (eds) Facets of vision. Springer Berlin Heidelberg, Berlin, Heidelberg, pp 152-172

Stavenga DG, Numan JAJ, Tinbergen J, Kuiper JW (1977) Insect pupil mechanisms. J Comp Physiol 113:73-93. https://doi.org/10.1007/bf00610454

Stavenga DG, Zantema A, Kuiper JW (1973) Rhodopsin processes and the function of the pupil mechanism in flies. In: Langer $\mathrm{H}$ (ed) Biochemistry and physiology of visual pigments. Springer Berlin Heidelberg, Berlin, Heidelberg, pp 175-180

Stein RB, French AS, Holden AV (1972) The frequency response, coherence, and information capacity of two neuronal models. Biophys J 12:295-322. https://doi.org/10.1016/S0006-3495(72)86087-9

Streck P (1972) Der Einfluß des Schirmpigmentes auf das Sehfeld einzelner Sehzellen der Fliege Calliphora erythrocephala Meig. Z Vgl Physiol 76:372-402. https://doi.org/10.1007/bf00337781

Walcott B (1971) Cell movement on light adaptation in the retina of Lethocerus (Belostomatidae, Hemiptera). Z Vgl Physiol 74:1-16. https://doi.org/10.1007/bf00297785

Ward CL, Hammen CS (1957) New mutations affecting tryptophan-derived eye pigments in three species of insects. Evolution 11:60-64

Wu CF, Wong F (1977) Frequency characteristics in the visual system of Drosophila: genetic dissection of electroretinogram components. J Gen Physiol 69:705-724 
Zhu H, Kirschfeld K (1984) Protection against photodestruction in fly photoreceptors by carotenoid pigments. J Comp Physiol A 154:153-156. https://doi.org/10.1007/bf00604980

Zhukovskaya M, Novikova E, Saari P, Frolov RV (2017) Behavioral responses to visual overstimulation in the cockroach Periplaneta americana L. J Comp Physiol A 203:1007-1015. https://doi.org/10.1007/s00359-017-1210-8 